not yet available to explain the aetiology of this disorder.

ARYA, D. K. (1991) Anorexia nervosa across cultures. British Journal of Psychiairy, 158, 285-286.

BRYANT-WAUGH, R. \& LASK, B. (1991) Anorexia nervosa in a group of Asian children living in Britain. British Journal of Psychiatry. 158, 229-233.

Hatrield, A. B., Spaniol, L. \& Zipple, A. M. (1987) Expressed emotions: a family perspective. Schizophrenia Bulletin, 13, 221-226.

Department of Psychiatry

Dinesh K. Arya

Queens Medical Centre

Nottingham NG7 2UH

\section{Abreaction before ECT}

SIR: We would agree with the general point made by Sivakumar et al (Journal, September 1991, 159, 444445), who suggest that the drug-assisted interview (DAI) is a procedure deserving of more attention, but would make a number of observations.

Sivakumar et al refer to 'abreactive techniques' as having 'evaluative and therapeutic' potential. DAIs have their roots in both biological psychiatry (narcosis therapies for psychotic disorders (Bleckwenn, 1930)) and dynamic psychiatry (drug assisted 'talking cures', generally based on early cathartic models (Horsley, 1936)). Most therapeutic applications of DAIs are related to these latter psychodynamic approaches to treatment. As we have pointed out, however, the status of abreaction - the free expression or release of previously repressed emotion within psychotherapy has been debated over many years (Patrick \& Howells, 1990). Although results may be dramatic, it is argued that alone it is insufficient to produce lasting change, passing over real areas of difficulty (Bronner, 1955). We think it has been correctly suggested that psychotherapeutic practice involves establishing a relationship in which trust, talk and understanding help a person to accept difficult issues, for which the patient needs to be fully conscious and cooperative (Brown \& Pedder, 1979). In addition, several studies confirm that, in the treatment of hysteria, DAIs produce no significantly better results than general psychiatric treatment, although in some instances there may be economy of time (Lambert \& Rees, 1944). Thus we would argue that valid therapeutic indications are few.

We believe that the mixture of biological and dynamic approaches implied within the term 'abreactive techniques' merely perpetuates uncertainty concerning the underlying intentions and indications of the interview. The term, 'drug-assisted interview' avoids such confusion.
Is there then a place for drug-assisted diagnostic interviews in clinical practice? A cautious appraisal of the literature suggests that the DAI may be useful in accessing the inaccessible patient and in the evaluation of disorders with a potential organic aetiology (Dysken et al, 1979). In disorders such as stupor, DAIs are no substitute for rigorous examination and investigation (Rashkin \& Frank, 1974). They may prove very useful however; such interviews often accentuate apparent cognitive impairment if the aetiology is organic (Weinstein et al, 1953), and may lead to dramatic improvement in functional disorders.

This rule may mislead. Not all patients with organic disease worsen under amylobarbitone, and certain organic disturbances caused by organic states, such as substance withdrawal and epilepsy, may be dramatically alleviated (Ward et al, 1978). In contrast, if insufficient drug is given, the lack of mental clearing may be falsely ascribed to the presence of organic pathology.

BLECKWENN, W. J. (1930) Production of sleep and rest in psychotic cases. Archives of Neurology and Psychiatry. 24, 365-372.

BronNER, A. (1955) The role of sodium amytal in psychotherapy and diagnosis. American Journal of Psychiatry, 9, 234-242.

Brown, D. \& PEDDER, J. (1979) Introduction to Psychotherapy. Tavistock: London.

Dysken, M. W., Chang, S. S., CASPER, R. C., et al (1979)

Barbiturate-facilitated interviewing. Biological Psychiatry, 14, $421-432$.

HoRSLEY, J. S. (1936) Narco-analysis. Lancet, i, 55-56.

A full list of references is available from the author.

Institute of Psychiatry

ROGER HOWELLS

De Crespigny Park

London SE5 $8 A Z$

Tavistock Clinic

MatTheW Patrick

120 Belsize Lane

London NW3 5DA

Special medical and nursing care needs of people with severe learning difficulties

SIR: The large hospitals for mental handicap usually had an infirmary ward for seriously and terminally ill patients. 'Demedicalisation' and 'normalisation' philosophies have prompted the closure of such provision in the belief that mentally handicapped people should be nursed in their own residences or, if they need intensive care, in general hospitals.

In practice, general hospitals are unable to give long-term nursing to people with severe learning difficulties beyond investigation, assessment, diagnosis and treatment of their illnesses. In Leeds, 